\title{
Psychiatric Presentation of Frontotemporal Dementia Associated with Inclusion Body Myopathy due to the VCP Mutation $(\mathrm{R} 155 \mathrm{H})$ in a French Family
}

\author{
Agnès Jacquin $^{\mathrm{a}, \mathrm{b}} \quad$ Olivier Rouaud $^{\mathrm{a}}$ Pierre Soichot $^{\mathrm{b}} \quad$ Yannick Bejot $^{\mathrm{a}}$ \\ Inna Dygai-Cochet ${ }^{c}$ Marie Sarazin ${ }^{d}$ Tania Stojkovic $^{\mathrm{e}}$ \\ Martine Lemesle-Martin ${ }^{b}$ Maurice Giroud ${ }^{a, b}$ Thibault Moreau $^{a, b}$ \\ ${ }^{a}$ Memory Centre, Department of Neurology, ${ }^{b}$ Neurophysiology, Department of Neurology, \\ and 'Department of Nuclear Medicine, Centre Georges François Leclerc, University \\ Hospital, Dijon, ${ }^{d}$ Memory Centre, Department of Neurology, and ${ }^{\mathrm{e} I n s t i t u t ~ d e ~ m y o l o g i e, ~}$ \\ Centre de références des affections neuromusculaires, Hôpital de la Pitié-Salpêtrière, \\ AP-HP, Paris, France
}

\section{Key Words}

Frontotemporal dementia - Inclusion body myopathy - VCP mutation - Psychiatric disorders

\begin{abstract}
Introduction: Inclusion body myopathy with Paget's disease of the bone and frontotemporal dementia (IBMPFD) is a rare late-onset autosomal dominant disorder due to a mutation of the valosin-containing protein (VCP) gene. Case Report: We report the case of a patient who developed progressive weakness of the limbs in his fifties, until he was confined to a wheelchair. At that time, he developed acute behavioural changes including irritability, severe anxiety and major depression, which led to him being hospitalised in a psychiatric hospital. $\mathrm{He}$ also suffered from aphasia and executive function impairment, which helped us to diagnose a behavioural form of frontotemporal dementia (FTD). The diagnosis of IBMPFD due to a mutation in the VCP gene was confirmed by a genetic study of the VCP gene (R155H mutation). Discussion: The clinical diagnosis of IBMPFD is suggested by the presence of at least one of three major manifestations as follows: inclusion body myopathy (mean onset at 42 years of age), Paget's disease of the bone and FTD (mean onset at 55 years of age). It is mostly the behavioural form of FTD (behavioural changes, executive dysfunction
\end{abstract}

Agnès Jacquin

Memory Centre, Dijon Stroke Registry (EA 4184)

Department of Neurology, University Hospital

3 Rue du Faubourg Raines, FR-21000 Dijon (France)

E-Mail agnesjacquin@free.fr 
and aphasia). One interesting finding in our report is the predominance of the psychiatric symptoms at the beginning of the behavioural changes, which led to the diagnosis of FTD. The diagnosis of IBMPFD was confirmed by the genetic study: the R155H mutation found on exon 5 domain CDC48 is the most frequent of the 18 known mutations in the VCP gene.

(c) 2013 S. Karger AG, Basel

\section{Introduction}

Inclusion body myopathy with Paget's disease of the bone and frontotemporal dementia (IBMPFD) is a rare late-onset autosomal dominant multi-system disorder, with variable penetrance of three typical clinical components: inclusion body myopathy, Paget's disease and frontotemporal dementia (behavioural form). This disorder is due to mutations of the valosin-containing protein (VCP) gene on chromosome 9p21-p12 [1, 2]. Eighteen mutations in 12 loci of the VCP gene have been identified, originally in North American families [3]. We report a French family carrying the R155H mutation of the VCP gene. The proband was diagnosed with inclusion body myopathy and then frontotemporal dementia with a psychiatric onset. We describe here the clinical and genetic features of IBMPFD.

\section{Case Report}

The proband was a 54-year-old French male patient with a personal history of dyslipidaemia and tuberculosis in childhood. From the age of 41 years, he slowly developed progressive weakness and atrophy of proximal upper-limb muscles and later (at 46 years of age) involvement of the lower limbs, associated with axial weakness. At that time, the walking test was unlimited, whereas at 50 years of age, he was only able to walk $50 \mathrm{~m}$ using a cane for support.

Then, the proband developed acute behavioural changes including irritability, severe anxiety and major depression, which led to him being hospitalised in a psychiatric hospital at 53 years of age. He developed gradual cognitive decline characterised by impairment of executive functions. The deterioration of upper- and lower-limb weakness and cognitive functions accelerated. The patient was eventually unable to walk and was confined to a wheelchair. His cognitive dysfunctions consisted of impaired frontal lobe functions, dominated by major behavioural changes (such as changes in affects, decline in social interpersonal conduct and perseveration) and aphasia (phonemic and semantic paraphasia). His score on the Frontotemporal Behavioural Scale was abnormal (3 items out of 4 were pathological). However, his memory was not impaired: the Mini-Mental State Examination (MMSE) was normal (29 out of 30 points).

An electromyography (EMG) (fig. 1) showed fibrillation potentials and positive sharp waves at rest, and myopathic or neurogenic features according to the muscles examined: polyphasic small-amplitude short-duration motor unit potentials and polyphasic longduration with slow-frequency motor unit potentials were found together in the EMG study. These results suggested inclusion body myopathy, which was confirmed by a muscle biopsy revealing histopathological changes consisting of rimmed vacuoles. Plasma creatine kinase levels were mildly increased (262 IU/l, normal values 20-220 IU/l). Nerve conduction studies were normal.

Cerebral magnetic resonance imaging (MRI) was performed at 54 years of age and revealed frontal and internal temporal atrophy (fig. 2). Fluorodeoxyglucose (FDG) positron 
emission tomography (PET) scan showed severe bilateral hypometabolism, involving especially the frontal and temporal lobes (fig. 3). The occipital lobe, basal ganglia and cerebellar metabolism were normal. Behavioural-type frontotemporal dementia was therefore diagnosed based on the clinical frontal lobe dysfunction and the results of the cerebral MRI and PET scan.

Moreover, the mother and the grandfather of the index patient had died with an unknown myopathy (confined to a wheelchair at the end of their lives). His mother also suffered from dementia. The index patient has an asymptomatic 25-year-old son.

The diagnosis of IBMPFD due to a mutation in the VCP gene was suggested given the personal and familial history of the patient. This diagnosis was confirmed by the genetic study of the VCP gene which revealed the VCP R155H mutation (exon 5, domain CDC48, 464 $\mathrm{G}>\mathrm{A}$ ). The genetic study was not performed in the mother and the grandfather of the index patient.

The absence of bone pain, hearing problems or spontaneous fractures, the normality of the serum concentration of alkaline phosphatase and the normality of a $99 \mathrm{mTC}$ total-body bone scan ruled out Paget's disease of the bone. The cardiac exam was normal.

\section{Discussion}

IBMPFD is a rare disorder originally described in North American families. Unlike hereditary inclusion body myopathy, which is autosomal recessive, its transmission is autosomal dominant.

The clinical diagnosis of IBMPFD is suggested by the presence of at least one of three major manifestations as follows $[1,2]$ : inclusion body myopathy, Paget's disease of the bone and frontotemporal dementia. Inclusion body myopathy is characterised by a mean onset at 42 years of age with progressive proximal and then distal muscle weakness and atrophy. EMG of affected muscles reveals fibrillations and positive sharp waves at rest, and an association with myopathic and neuropathic potentials with movement. Plasma creatine kinase levels are mostly normal or mildly increased. Frontotemporal dementia begins at 55 years of age on average and is characterised by the predominance of behavioural changes (disinhibition, irritability and anxiety), executive dysfunction and aphasia; the memory is preserved for a long time. One interesting finding in our case report is the onset of frontotemporal dementia in the form of psychiatric symptoms, which has rarely been described in previous studies $[3,4]$. The diagnosis of frontotemporal dementia is confirmed, on the one hand, by frontal and temporal atrophy on cerebral MRI and, on the other hand, by frontal and temporal hypometabolism on the FDG PET scan [5]. In our case, frontotemporal hypometabolism on the PET scan seemed to be more severe than expected with atrophy in these areas on MRI. In fact, the higher severity of hypometabolism compared to atrophy in the same areas of the brain suggests some functional alteration such as disruption of connected areas [6]. Bone involvement is typical of Paget's disease, which also appears at 42 years of age on average, with raised serum concentrations of alkaline phosphatase and abnormalities on bone scintigraphy. However, the penetrance of IBMPFD is variable according to the type of mutation of the VCP gene and is age-related. Only $12 \%$ of the patients with IBMPFD described in the literature had all the three major manifestations of the disease previously described; $50 \%$ had only two major manifestations in any combination $(20 \%$ had inclusion body myopathy associated with frontotemporal dementia as did our proband). Other phenotypic features have been reported, including dilated cardiomyopathy, hepatic fibrosis, cataracts and sensory-motor axonal neuropathy [2]. 
At muscle biopsy, the paucity of abnormalities suggesting hereditary inclusion body myopathy associated with a mutation of the VCP gene is frequent, even when this is performed on an affected muscle: in our case, only rimmed vacuoles, which are non-specific neuropathological disorders, suggestive of both sporadic and hereditary inclusion body myopathy, were found at biopsy. In fact, whereas IBMPFD muscle biopsies have variable myopathic features, immunohistochemical analyses mostly find inclusions of mutant VCP, which co-localise with accumulations of ubiquitinated proteins and TDP-43 (TAR DNAbinding protein 43) inclusions and are more specific to the hereditary association between inclusion body myopathy and frontotemporal dementia [2, 7]. These neuropathological disorders are suggestive of an alteration of the ubiquitin-proteasome system.

Concerning the genetic study of our proband, the R155H mutation found on exon 5, domain CDC48, in the VCP gene corresponds to one of the mutations originally described in North American families [1]. This mutation has recently been discovered in an Italian family [8]. Eighteen mutations in 12 loci of the VCP gene in the chromosome 9p21-p12 have been identified and are listed in table 1 . The genetic studies were not performed for the asymptomatic index patient's son. The phenotypes are inconsistent with the different VCP mutations: in most cases, the onset of Paget's disease of the bone in patients with the R155H mutation, as carried by our index patient, occurs later than is the case with other VCP mutations. As a consequence, we cannot exclude the possibility that he will develop Paget's disease of the bone later.

To conclude, even though IBMPFD is a rare clinical entity, the diagnosis should be considered when a patient suffers from inclusion body myopathy or Paget's disease of the bone or frontotemporal dementia and has a similar family history. Frontotemporal dementia occurs more often in a behavioural form and can begin with psychiatric symptoms alone. Known or new mutations of the VCP gene should be screened for in order to confirm the suspected clinical diagnosis.

\section{Acknowledgements}

We thank Philip Bastable for English language reviewing.

\section{Disclosure Statement}

The authors have no conflicts of interest.

\section{References}

1 Watts G, Wymer J, Kovach M, et al: Inclusion body myopathy associated with Paget disease of the bone and frontotemporal dementia is caused by mutant valosin-containing protein. Nat Genet 2004;36:377-381.

2 Weihl C, Pestronk A, Kimonis V: Valosin-containing protein disease: inclusion body myopathy with Paget's disease of the bone and frontotemporal dementia. Neuromusc Disord 2009;19:308-315.

3 Kimonis V, Fulchiero E, Vesa J, et al: VCP disease associated with myopathy, Paget disease of bone and frontotemporal dementia: review of a unique disorder. Biochimica et Biophysica Acta 2008;1782:744-748.

4 Stojkovic T, Hammouda EH, Richard P, et al: Clinical outcome in 19 French and Spanish patients with valosin-containing protein myopathy associated with Paget's disease of bone and frontotemporal dementia. Neuromusc Disord 2009;19:316-323.

5 Rohrer JD: Structural brain imaging in frontotemporal dementia. Biochimica et Biophysica Acta 2012;1822:325-332. 
Jacquin et al.: Psychiatric Presentation of Frontotemporal Dementia Associated with Inclusion Body Myopathy due to the VCP Mutation (R155H) in a French Family

6 Chételat G, Desgranges B, Landeau B, et al: Direct voxel-based comparison between grey matter hypometabolism and atrophy in Alzheimer's disease. Brain 2008;131:60-71.

7 Weihl C, Temiz P, Miller S, et al: TDP-43 accumulation in IBM muscle suggests a common pathogenic mechanism with frontotemporal dementia. J Neurol Neurosurg Psychiatry 2008;79:1186-1189.

8 Viassolo V, Previtali SC, Schiatti E, et al: Inclusion body myopathy, Paget's disease of the bone and frontotemporal dementia: recurrence of the VCP R155H mutation in an Italian family and implications for genetic counselling. Clin Genet 2008;74:54-60.

-9 Guyant-Maréchal L, Laquerrière A, Duyckaerts C, et al: Valosin-containing protein gene mutations. Neurology 2006;67:644-651.

$\$ 10$ Schröder R, Watts GD, Mehta SG, et al: Mutant valosin-containing protein causes a novel type of frontotemporal dementia. Ann Neurol 2005;57:457-461.

11 Gidaro T, Modoni A, Sabatelli M, et al: An Italian family with inclusion-body myopathy and frontotemporal dementia due to mutation in the VCP gene. Muscle Nerve 2008;37:111-114.

$\checkmark 12$ Kumar K, Needham M, Mina K, et al: Two Australian families with inclusion-body myopathy, Paget's disease of bone and frontotemporal dementia: novel clinical and genetic findings. Neuromusc Disord 2010;20:330334.

13 Djamshidian A, Schaeffer J, Haubenberger D, et al: A novel mutation in the VCP gene (G157R) in a German family with inclusion-body myopathy with Paget disease of bone and frontotemporal dementia. Muscle Nerve 2009;39:389-391.

14 Haubenberger D, Bittner RE, Rauch-Shorny S, et al: Inclusion body myopathy and Paget disease is linked to a novel mutation in the VCP gene. Neurology 2005;65:1304-1305.

15 Bersano A, Del Bo R, Lamperti C, et al: Inclusion body myopathy and frontotemporal dementia caused by a novel VCP mutation. Neurobiol Aging 2009;30:752-758.

Table 1. Mutations of the VCP gene currently identified in the literature

\begin{tabular}{lllll}
\hline Amino acid & Base change & Exon & Domain & References \\
\hline R93C & $277 \mathrm{C}>\mathrm{T}$ & 3 & CDC 48 & {$[9]$} \\
R95G & $283 \mathrm{C}>\mathrm{G}$ & 3 & CDC 48 & {$[1]$} \\
R95C & $283 \mathrm{C}>\mathrm{T}$ & 3 & CDC 48 & {$[3]$} \\
P137L & $410 \mathrm{C}>\mathrm{T}$ & 4 & CDC 48 & {$[4]$} \\
R155C & $463 \mathrm{C}>\mathrm{T}$ & 5 & CDC 48 & {$[1,4,9-11]$} \\
R155H & $464 \mathrm{G}>\mathrm{A}$ & 5 & CDC 48 & {$[1,4,8,9]$} \\
R155L & $464 \mathrm{G}>\mathrm{T}$ & 5 & CDC 48 & {$[12]$} \\
R155P & $464 \mathrm{G}>\mathrm{C}$ & 5 & CDC 48 & {$[1]$} \\
R155S & $463 \mathrm{C}>\mathrm{A}$ & 5 & CDC 48 & {$[4]$} \\
G157R & $469 \mathrm{G}>\mathrm{C}$ & 5 & CDC 48 & {$[4,13]$} \\
R159H & $476 \mathrm{G}>\mathrm{A}$ & 5 & CDC 48 & {$[4,14]$} \\
R159C & $476 \mathrm{G}>\mathrm{A}$ & 5 & CDC 48 & {$[15]$} \\
R191Q & $572 \mathrm{G}>\mathrm{A}$ & 5 & Linker 1 & {$[1,4]$} \\
L198W & $593 \mathrm{~T}>\mathrm{G}$ & 6 & Linker 1 & {$[12]$} \\
A232E & $695 \mathrm{C}>\mathrm{A}$ & 6 & Junction (L1-D1) [1] \\
T262A & N.A. & 7 & AAA D1 & {$[3]$} \\
N387H & $1159 \mathrm{~A}>\mathrm{C}$ & 10 & AAA D1 & {$[3]$} \\
A439S & $1315 \mathrm{G}>\mathrm{T}$ & 11 & AAA D1 & {$[4]$} \\
\hline
\end{tabular}

N.A. $=$ Not available. 
Jacquin et al.: Psychiatric Presentation of Frontotemporal Dementia Associated with Inclusion Body Myopathy due to the VCP Mutation (R155H) in a French Family
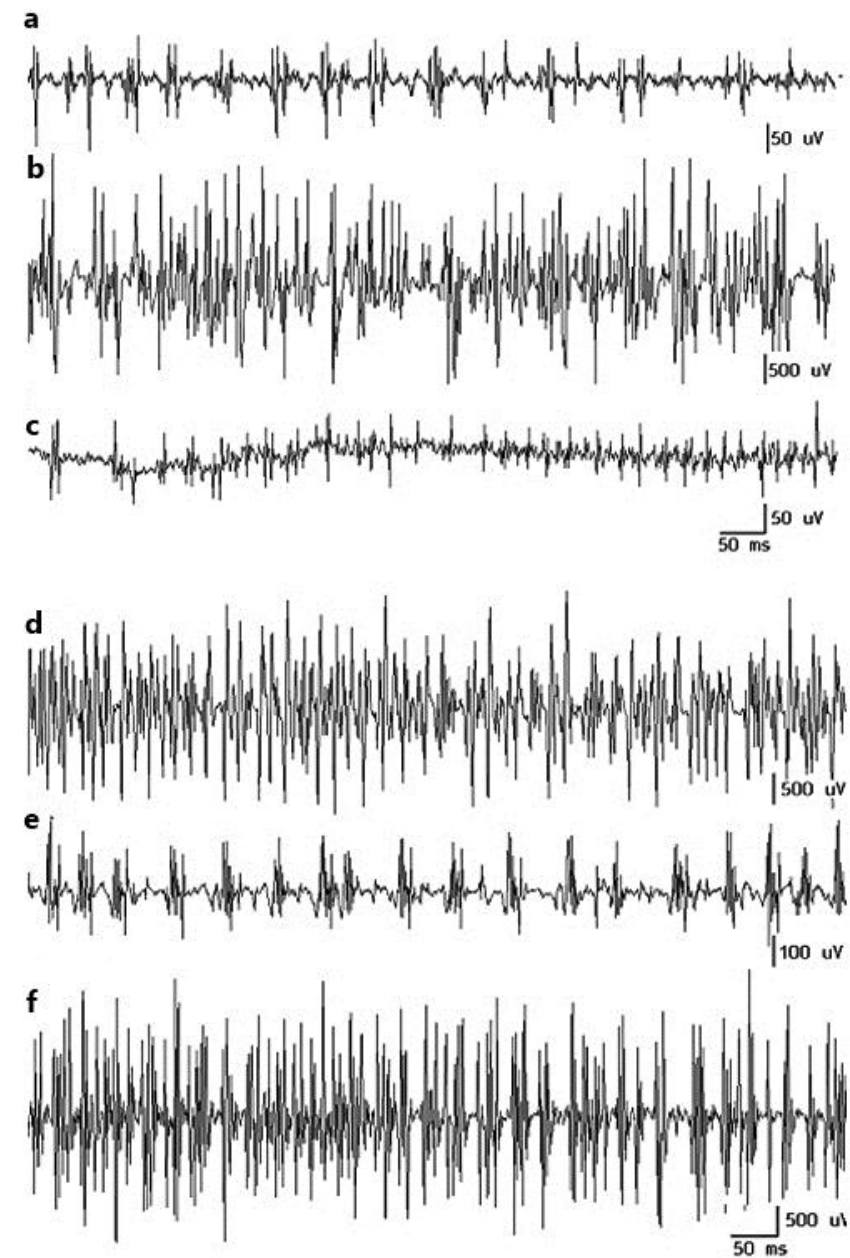

Fig. 1. EMG of the upper and lower limbs of our patient. a EMG of the left biceps brachii muscle: manual muscle testing $2 / 5$, fibrillation potentials and positive sharp waves at rest, polyphasic small-amplitude short-duration motor unit potentials with movement (myopathic potentials). b EMG of the left flexor digitorum sublimis muscle: manual muscle testing $4 / 5$, absence of potentials or positive sharp waves at rest, polyphasic high-amplitude and high-frequency potentials with movement (neuropathic potentials). c EMG of the left flexor digitorum profundus muscle: manual muscle testing 2/5, fibrillation potentials and positive sharp waves at rest, polyphasic small-amplitude short-duration motor unit potentials with movement (myopathic potentials). d EMG of the left vastus lateralis muscle: manual muscle testing 3/5, fibrillation potentials and positive sharp waves at rest, polyphasic high-amplitude and high-frequency potentials with movement (neuropathic potentials). e EMG of the left tibialis anterior muscle: manual muscle testing $1 / 5$, severe fibrillation potentials and positive sharp waves at rest, rare polyphasic smallamplitude short-duration motor unit potentials with movement (myopathic potentials). $f$ EMG of the left extensor digitorum brevis muscle: manual muscle testing $4 / 5$, absence of potentials or positive sharp waves at rest, polyphasic high-amplitude and high-frequency potentials with movement (neuropathic potentials). 
Jacquin et al.: Psychiatric Presentation of Frontotemporal Dementia Associated with Inclusion Body Myopathy due to the VCP Mutation (R155H) in a French Family

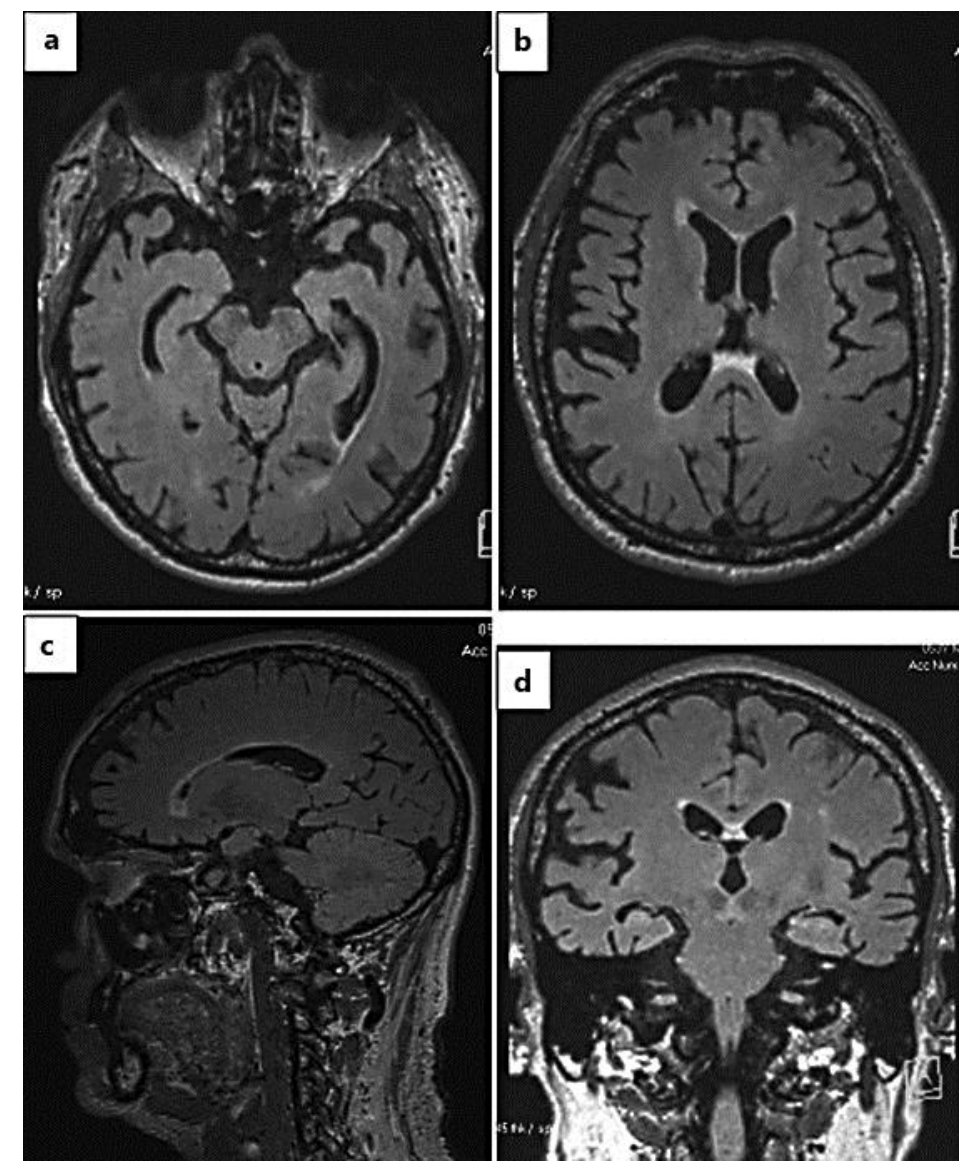

Fig. 2. T2-FLAIR-weighted brain MRI of our proband. a Axial image throughout the hippocampal structures: bilateral temporal atrophy. b Axial image throughout the frontal lobes: bilateral frontal atrophy. c Sagittal image: frontal atrophy. d Coronal image: bilateral temporal atrophy. 
Jacquin et al.: Psychiatric Presentation of Frontotemporal Dementia Associated with Inclusion Body Myopathy due to the VCP Mutation (R155H) in a French Family

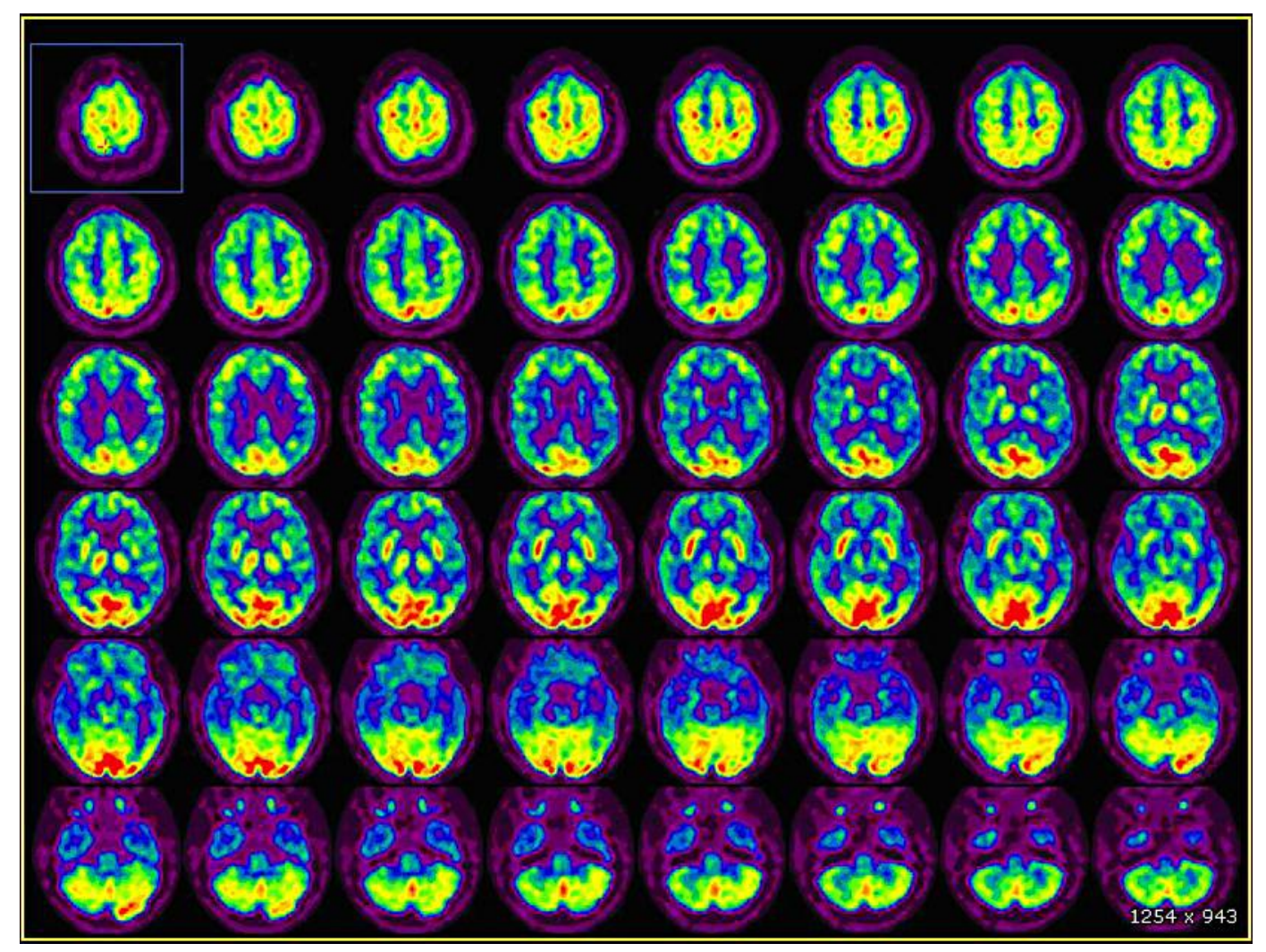

Fig. 3. FDG PET scan in our proband: severe bilateral hypometabolism, involving especially the frontal and temporal lobes. 